\title{
Uso do Coeficiente Alfa de Cronbach na Validação de Questionários Utilizados na Pesquisa Odontológica.
}

\author{
Cronbach alfa coeficient use for validation of questionnaires in dental \\ research.
}

\author{
Rafael Gustavo Dal Moro* \\ Melissa Rosa Braga** \\ Dalva Maria Pereira Padilha***
}

\begin{abstract}
RESUMO
Questionários são freqüentemente utilizados na pesquisa odontológica. A validação dos instrumentos de coleta de dados é necessária para a análise dos resultados. A checagem da consistência interna é um dos passos da validação, podendo ser realizada através do cálculo do coeficiente alfa de Cronbach. 0 objetivo deste estudo é demonstrar o cálculo do coeficiente de consistência interna proposto por Cronbach. Um questionário respondido por 51 idosos, sobre conhecimento em higiene bucal, foi utilizado. Uma vez que o coeficiente obtido foi igual a 0,72 , pode-se concluir que o presente instrumento possui boa consistência interna. A confiabilidade dos dados é resultado da qualidade dos instrumentos de coleta de dados. Sendo assim é importante validar estes instrumentos, sendo a checagem da consistência interna uma das etapas da validação.
\end{abstract}

PALAVRAS-CHAVE

Coleta de dados, Validade de resultados

\section{INTRODUÇÃ O}

O conhecimento científico é resultado de uma investigação sistemática e metódica da realidade, buscando descobrir as causas dos fenômenos e suscitar leis generalizadas que os regem. $\mathrm{O}$ trabalho científico é composto por etapas sistemáticas e orientadas para alcançar os objetivos propostos (ESTRELA; SABINO, 2001).

Após definir os objetivos, no trabalho deve constar suas etapas metodológicas. Um dos passos da metodologia é a coleta de dados. Sua realização depende de um instrumento, seja um questionário, entrevistas ou um exame clínico. Questionários são amplamente utilizados quando se deseja avaliar conhecimentos e atitudes sobre determinado assunto (ESTRELA; SABINO, 2001).

A elaboração de questionário deve ser feita de maneira cuidadosa. Questionários construídos para uma determinada faixa etária provavelmente não funcionarão bem em outros grupos etários. Todo instrumento de coleta de dados é desenvolvido com um propósito, possuindo pontos fortes e fracos. Alguns instrumentos fornecem respostas precisas, contudo são longos e complexos. Outros são mais simples, rápidos e capazes de estimar a capacidade global, entretanto sem investigar os detalhes do assunto em questão(CRONBACH, 1996).

A utilização de questionários como instrumento de coleta de dados gera dúvidas sobre sua validade, ou seja, se o instrumento realmente está medindo o que se tem a intenção de medir (ABEGG, 2002). Uma das etapas da validação de um questionário é a checagem de sua consistência interna. De maneira geral podese definir a consistência interna como a avaliação da homogeneidade dos itens, ou seja, se os itens ou perguntas do questionário avaliam de maneira semelhante aquilo a que se propõe a avaliar (CRONBACH, 1996).

O cálculo do coeficiente alfa de Cronbach é um dos procedimentos mais utilizados na avaliação da consistência interna, denominado como coeficiente de fidedignidade para uma média ou total de itens avaliados em uma amostra (CRONBACH, 1996).

O presente trabalho tem como objetivo demonstrar o cálculo do coeficiente alfa de Cronbach em um questionário utilizado em uma pesquisa na área odontológica.

\section{METODOLOGIA}

O questionário foi aplicado em uma amostra de 51 idosos institucionalizados com o objetivo de avaliar conhecimento sobre higiene bucal. Julgou-se apropriado elaborar um instrumento de rápida aplicação e fácil compreensão para os idosos. Desta forma, optou-se por formular 10 questões com respostas do tipo sim ou não. Itens julgados corretamente recebem escore 1 e julgados incorretamente recebem escore 0 . $\mathrm{O}$ escore geral do instrumento pode variar de 0 a 10 para cada participante.

O procedimento de validação iniciou- se aplicando o instrumento em 10 idosos que em seguida foram entrevistados quanto às dúvidas no entendimento e clareza das questões, bem como do grau de dificuldade das perguntas.

A segunda etapa da validação do questionário é a verificação da consistência interna através do cálculo do coeficiente de Cronbach.

A seguinte fórmula é proposta para o cálculo do coeficiente (CRONBACH, 1996), sendo:

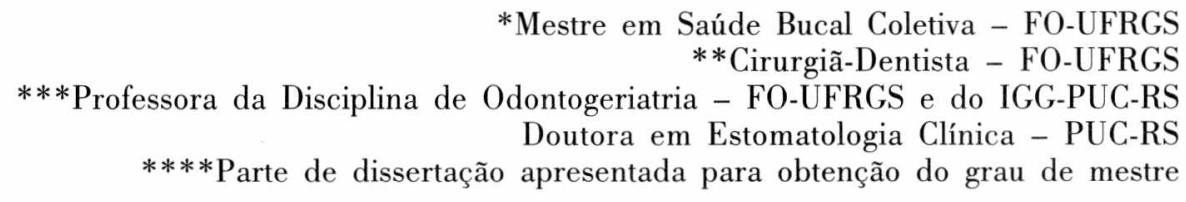


A seguinte fórmula é proposta para o cálculo do coeficiente (CRONBACH, 1996), sendo:

$\mathrm{k}=$ número de itens do questionário

$\sum s_{\text {itens }}^{2}=$ somatório da variância dos itens do questionário

$s_{\text {total }}^{2}$ variância do escore total do questionário

$$
\alpha=\frac{k}{k-1}\left[1-\frac{\sum s_{\text {itens }}^{2}}{s_{\text {total }}^{2}}\right]
$$

A variância por sua vez é calculada através da seguinte fórmula (ALTMAN, 1991), sendo:

- $\mathrm{x}=$ escore para cada indivíduo

$\bar{x}=$ média do escore

n $=$ número de observações (participantes)

$$
s^{2}=\frac{\sum(x-\bar{x})^{2}}{n-1}
$$

\section{RESULTADOS}

A tabela 1 apresenta dados parciais sobre os escores por item e escore total para cada paciente submetido ao questionário.

Substituindo os valores da tabela 1 na fórmula apresentada para o cálculo do coeficiente. obtêm-se:

$\alpha=\frac{10}{10-1}\left[1-\frac{\sum(0,21+0,24+0,25+0,25+0,18+\ldots 0,25)}{6,29}\right]$

$$
\begin{gathered}
\alpha=1,11\left[1-\frac{2,21}{6,29}\right] \\
\alpha=1,11,0,65 \\
\alpha=0,72
\end{gathered}
$$

Tabela 1. Dados obtidos através do teste de conhecimento sobre higiene bucal.

\section{Escore por item}

Item

Escore total

$\begin{array}{llllllllllll}\text { lf } & 1 & 2 & 3 & 4 & 5 & 6 & 7 & 8 & 9 & 10 & \text { do teste }\end{array}$

$\begin{array}{lllllllllllll}\text { Paciente } & 1 & 0 & 0 & 0 & 1 & 0 & 0 & 0 & 0 & 0 & 0 & 1\end{array}$

$\begin{array}{lllllllllllll}\text { Paciente } & 2 & 0 & 0 & 0 & 0 & 0 & 0 & 0 & 1 & 0 & 0 & 1\end{array}$

$\begin{array}{lllllllllllll}\text { Paciente } & 3 & 0 & 0 & 0 & 0 & 1 & 0 & 1 & 0 & 0 & 0 & 2\end{array}$

$\begin{array}{lllllllllllll}\text { Paciente } & 4 & 0 & 0 & 1 & 1 & 0 & 0 & 0 & 0 & 0 & 0 & 2\end{array}$

$\begin{array}{lllllllllllll}\text { Paciente } & 5 & 1 & 0 & 0 & 0 & 0 & 0 & 0 & 0 & 0 & 1 & 2\end{array}$

$\begin{array}{lllllllllllll}\text { Paciente } & 6 & 0 & 0 & 1 & 0 & 0 & 0 & 0 & 1 & 0 & 0 & 2\end{array}$

$\begin{array}{lllllllllllll}\text { Paciente } & 7 & 0 & 0 & 0 & 0 & 1 & 0 & 1 & 0 & 0 & 0 & 2\end{array}$

$\begin{array}{lllllllllllll}\text { Paciente } & 8 & 0 & 0 & 1 & 0 & 1 & 0 & 0 & 1 & 0 & 0 & 3\end{array}$

$\begin{array}{lllllllllllll}\text { Paciente } & 9 & 0 & 0 & 1 & 0 & 0 & 1 & 0 & 0 & 1 & 0 & 3\end{array}$

$\begin{array}{lllllllllllll}\text { Paciente } & 10 & 0 & 1 & 0 & 1 & 0 & 0 & 1 & 0 & 0 & 0 & 3\end{array}$

$\begin{array}{lllllllllllll}\text { Paciente } & 11 & 1 & 0 & 0 & 0 & 1 & 1 & 0 & 0 & 0 & 0 & 3\end{array}$

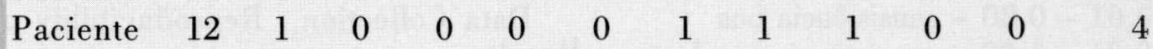

$\begin{array}{lllllllllllll}\text { Paciente } & 13 & 0 & 1 & 0 & 1 & 1 & 0 & 1 & 0 & 0 & 0 & 4\end{array}$

$\begin{array}{lllllllllllll}\text { Paciente } & 14 & 1 & 1 & 0 & 0 & 1 & 0 & 0 & 1 & 0 & 0 & 4\end{array}$

$\begin{array}{lllllllllllll}\text { Paciente } & 15 & 1 & 0 & 0 & 1 & 0 & 1 & 1 & 0 & 0 & 0 & 4\end{array}$

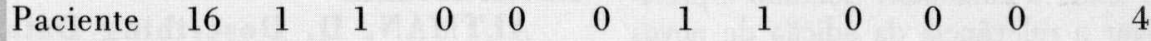

$\begin{array}{lllllllllllll}\text { Paciente } & 17 & 0 & 0 & 1 & 0 & 1 & 0 & 1 & 1 & 0 & 0 & 4\end{array}$

$\begin{array}{lllllllllllll}\text { Paciente } & 18 & 0 & 1 & 1 & 1 & 0 & 0 & 0 & 1 & 0 & 1 & 5\end{array}$

$\begin{array}{lllllllllllll}\text { Paciente } & 19 & 1 & 1 & 0 & 1 & 1 & 0 & 1 & 0 & 0 & 0 & 5\end{array}$

$\begin{array}{lllllllllllll}\text { Paciente } & 20 & 1 & 1 & 0 & 0 & 1 & 1 & 0 & 0 & 0 & 1 & 5\end{array}$

$\begin{array}{lllllllllllll}\text { Paciente } & 21 & 1 & 1 & 0 & 0 & 1 & 1 & 1 & 1 & 0 & 0 & 6\end{array}$

$\begin{array}{lllllllllllll}\text { Paciente } & 22 & 1 & 0 & 0 & 1 & 1 & 1 & 1 & 1 & 0 & 0 & 6\end{array}$

$\begin{array}{lllllllllllll}\text { Paciente } & 23 & 1 & 1 & 0 & 0 & 1 & 1 & 1 & 0 & 0 & 1 & 6\end{array}$

$\begin{array}{lllllllllllll}\text { Paciente } & 24 & 1 & 1 & 0 & 0 & 1 & 1 & 1 & 0 & 0 & 1 & 6\end{array}$

$\begin{array}{lllllllllllll}\text { Paciente } & 25 & 1 & 0 & 1 & 0 & 1 & 0 & 1 & 1 & 1 & 0 & 6\end{array}$

$\begin{array}{lllllllllllll}\text { Paciente } & 26 & 1 & 0 & 0 & 1 & 1 & 1 & 1 & 0 & 0 & 1 & 6\end{array}$

$\begin{array}{lllllllllllll}\text { Paciente } & 27 & 1 & 0 & 0 & 1 & 1 & 1 & 1 & 1 & 0 & 0 & 6\end{array}$

$\begin{array}{lllllllllllll}\text { Paciente } & 28 & 0 & 1 & 1 & 0 & 1 & 1 & 1 & 1 & 0 & 0 & 6\end{array}$

$\begin{array}{lllllllllllll}\text { Paciente } & 29 & 1 & 1 & 0 & 0 & 0 & 1 & 1 & 1 & 0 & 1 & 6\end{array}$

$\begin{array}{lllllllllllll}\text { Paciente } & 30 & 1 & 1 & 0 & 1 & 1 & 1 & 1 & 0 & 0 & 0 & 6\end{array}$

$\begin{array}{lllllllllllll}\text { Paciente } & 31 & 1 & 1 & 0 & 1 & 1 & 1 & 1 & 0 & 0 & 0 & 6\end{array}$

$\begin{array}{lllllllllllll}\text { Paciente } & 32 & 0 & 1 & 0 & 1 & 1 & 1 & 1 & 1 & 0 & 1 & 7\end{array}$

$\begin{array}{lllllllllllll}\text { Paciente } & 33 & 1 & 1 & 1 & 0 & 1 & 1 & 1 & 0 & 0 & 1 & 7\end{array}$

$\begin{array}{lllllllllllll}\text { Paciente } & 34 & 1 & 1 & 0 & 1 & 1 & 1 & 1 & 0 & 0 & 1 & 7\end{array}$

$\begin{array}{lllllllllllll}\text { Paciente } & 35 & 1 & 0 & 0 & 0 & 1 & 1 & 1 & 1 & 1 & 1 & 7\end{array}$

$\begin{array}{lllllllllllll}\text { Paciente } & 36 & 1 & 1 & 0 & 1 & 1 & 1 & 1 & 1 & 0 & 0 & 7\end{array}$

$\begin{array}{lllllllllllll}\text { Paciente } & 37 & 1 & 1 & 1 & 1 & 1 & 1 & 1 & 1 & 0 & 0 & 8\end{array}$

$\begin{array}{lllllllllllll}\text { Paciente } & 38 & 1 & 1 & 1 & 1 & 1 & 1 & 1 & 0 & 0 & 1 & 8\end{array}$

$\begin{array}{lllllllllllll}\text { Paciente } & 39 & 1 & 1 & 1 & 1 & 1 & 1 & 0 & 1 & 1 & 0 & 8\end{array}$

$\begin{array}{lllllllllllll}\text { Paciente } & 40 & 0 & 1 & 1 & 1 & 1 & 1 & 1 & 1 & 0 & 1 & 8\end{array}$

$\begin{array}{lllllllllllll}\text { Paciente } & 41 & 1 & 1 & 1 & 0 & 1 & 1 & 1 & 0 & 1 & 1 & 8\end{array}$

$\begin{array}{lllllllllllll}\text { Paciente } & 42 & 1 & 1 & 1 & 1 & 1 & 1 & 1 & 1 & 0 & 0 & 8\end{array}$

$\begin{array}{lllllllllllll}\text { Paciente } & 43 & 1 & 1 & 1 & 1 & 1 & 1 & 1 & 0 & 1 & 1 & 9\end{array}$

$\begin{array}{lllllllllllll}\text { Paciente } & 44 & 1 & 1 & 1 & 1 & 1 & 1 & 1 & 1 & 0 & 1 & 9\end{array}$

$\begin{array}{lllllllllllll}\text { Paciente } & 45 & 1 & 1 & 0 & 1 & 1 & 1 & 1 & 1 & 1 & 1 & 9\end{array}$

$\begin{array}{lllllllllllll}\text { Paciente } & 46 & 1 & 0 & 1 & 1 & 1 & 1 & 1 & 1 & 1 & 1 & 9\end{array}$

$\begin{array}{lllllllllllll}\text { Paciente } & 47 & 1 & 1 & 1 & 1 & 1 & 1 & 1 & 0 & 1 & 1 & 9\end{array}$

$\begin{array}{lllllllllllll}\text { Paciente } & 48 & 1 & 1 & 1 & 1 & 1 & 1 & 1 & 1 & 0 & 1 & 9\end{array}$

$\begin{array}{lllllllllllll}\text { Paciente } & 49 & 1 & 1 & 1 & 1 & 1 & 1 & 1 & 1 & 0 & 1 & 9\end{array}$

$\begin{array}{lllllllllllll}\text { Paciente } & 50 & 1 & 1 & 1 & 1 & 1 & 1 & 1 & 1 & 1 & 1 & 10\end{array}$

$\begin{array}{lllllllllllll}\text { Paciente } & 51 & 1 & 1 & 1 & 1 & 1 & 1 & 1 & 1 & 1 & 1 & 10\end{array}$

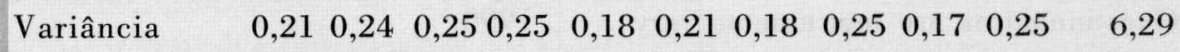




\section{DISCUSSÃO}

O uso de questionários é comum na pesquisa médica e odontológica. $O$ cálculo do coeficiente de Cronbach é indicado para avaliar a consistência interna de uma escala, também chamada de medida de homogeneidade inter-item. O coeficiente é influenciado em grande parte pela homogeneidade dos itens da escala bem como pelo número de itens presentes no instrumento.

Assim, quanto maior a homogeneidade dos itens, maior o coeficiente alfa. Sneed e Herman (1991) apontam os valores de referência para a interpretação do coeficiente:

- $<0,20$ - consistência fraca

- 0,21 - 0,40 - consistência regular

- 0,41 - 0,60 - consistência moderada

- 0,61 - 0,80 - consistência boa

- 0,81 - 1,00 - consistência excelente

Geralmente quando se aumenta o número de itens de um instrumento, o coeficiente tende a aumentar. Contudo é preciso julgar a relevância da adição de novos itens, uma vez o instrumento torna-se mais complexo e mais demorado de ser respondido (SNEED; HERMAN, 1991).

Recentemente Frazer e Lindsay (2001) desenvolveram um questionário para avaliar a preocupação com a aparência dos dentes. 0 instrumento contendo 42 itens foi aplicado em uma amostra de 100 adultos. O coeficiente alfa de Cronbach obtido foi de 0,90, indicando excelente consistência interna. Entretanto, poucos estudos sobre validação de questionários são encontrados na literatura odontológica.

Uma vez que a confiabilidade dos dados está diretamente ligada a qualidade dos instrumentos de coleta de dados, deve-se fazer uso de métodos e procedimentos de validação destes instrumentos, sendo a checagem da consistência interna uma das etapas da validação.

\section{CONCLUSÃO}

O presente estudo teve como objetivo demonstrar a utilização do coeficiente alfa de Cronbach na avaliação da consistência interna de um questionário elaborado em uma pesquisa odontológica. O coeficiente encontrado foi igual a 0,72 , indicando boa consistência interna. Este procedimento de validação é relativamente fácil de ser realizado e extremamente importante uma vez que auxilia na avaliação da qualidade de instrumentos de coleta de dados para a pesquisa odontológica.

\section{ABSTRACT}

Questionnaires are instruments very used frequently in dental research. The validity of data collection instruments is necessary for results analysis. Internal consistency evaluation is a step for validation. Cronbach alfa coeficient was developed for that calculation. The aim of this study is to demonstrate the calculation of the coeficient of internal consistency proposed by Cronbach. A questionnaire answered by 51 elderly people about oral hygiene knowledge was used. Once the alfa coeficient found was 0,72 , the instrument had good internal consistency. Data confiability is a result of the quality of data collection instruments, so it is important to validate this instrument also by internal consistency evaluation.

\section{KEYWORDS}

Data Collection, Reproducibility of Results

\section{REFERÊNCIAS}

ALTMAN, D. Describing Data. In: - Practical Statistics

for Medical Research. Padstow: Chapman \& Hall, 1991. Cap.3, p.19-47.

\begin{abstract}
ABEGG, C. Como Fazer Levantamento de Dados. In: BERNI, D. Técnicas de Pesquisa em Economia. São Paulo: Saraiva, 2002. Cap.5, p.133151.
\end{abstract}

CRONBACH, L. Como Julgar os Testes: Fidedignidade e Outras Qualidades.. In:

Fundamentos da

Testagem Psicológica Porto Alegre: Artes Medicas, 1996. Cap. 6, p. 176 201.

ESTRELA, C.; SABINO, G. Estruturação do Trabalho Científico. In: ESTRELA, C. Metodologia Científica: Ensino e Pesquisa em Odontologia. São Paulo: Artes Médicas, 2001. Cap. 7, p.99-120.

FRAZER, M.; LINDSAY, S. Development of a Questionnaire to Measure Concern for Dental Appearance. Psychol. Rep., Louisville, v.89, no.2, p.425-430, Oct. 2001.

SNEED J.; HERMAN C. Influence of Job Characteristics and Organizational Commitment on Job Satisfaction of Hospital Foodservice Employees. J. Am. Diet. Assoc., Chicago, v.90, no.8, p. 1072-1076, Aug. 1990.
Endereço para Correspondência: Rafael Gustavo Dal Moro Rua Julio Bocaccio, 80 apto. 303 Santo Antônio - Porto Alegre - RS CEP: 90640-020

E-mail: rgdalmoro@yahoo.com.br 